\title{
Computer Simulations of Planet Collisions Using a New Parallel Algorithm
}

\author{
W. Even and M.W. Roth \\ Department of Physics \\ University of Northern lowa \\ Cedar Falls, IA 50614-0150 USA
}

Received February 22, 2002 Accepted May 22, 2002

\begin{abstract}
New deterministic computer simulations have been developed for studying the dynamics of large objects colliding. For various initial conditions the system may clump together forming a new object or may rotate, causing ejecta to leave the primary mass in a pattern sustaining only a short number of orbits, but promising of satellite formation. Bodies without a large central mass are not able to form a cohesive object, and fly apart upon impact. A rudimentary scaling study when the code is parallelized using a force-decomposition scheme suggests that the computational time scales inversely as the number of processors when less than four are involved and the gains are somewhat less pronounced as the number of processors increases.
\end{abstract}

\section{INTRODUCTION}

The formation of planets and solar systems has been a question in the scientific community for many centuries. A variety of analytical and numerical approaches have been put forth in an attempt to explain various aspects of the sun, moon, and planets within the last twenty years. Just recently, however, science has been able to apply numerical methods and conduct computational investigations into the actual dynamics and stochastic processes believed to be active in planetary formation and change. Ring and satellite systems are of particular interest: are they formed during various stages in the early history of a planet or can they be formed by trauma to an already formed body? Whether planet and solar system dynamics are modeled from a fluid-dynamical approach or a manyparticle-system approach almost any study will contribute new insight and results to the field.

A fair amount of work has been done in an attempt to model the time evolution of the proto-solar system using hydrodynamic models with emphasis on circumstellar ring formation [1-7] and even ring formation using interacting gravitating particles $[8,9]$. Most closely related to the work presented here are the very interesting results of 36 smooth particle hydrodynamics (SPH) simulations of lunar formation by means of a large body impacting the Earth [10]. There were anywhere from 20,000 to 30,000 particles in the simulations and a wide range of initial conditions were examined. This work incorporates the Tilloston equation of state (EOS) originally developed to describe strong shock in condensed matter to model the various changes of phase that might occur throughout a collision between planets. The simulations incorporated a final angular momentum constrained to be within $10 \%$ of the current earth-moon angular momentum. Simulations with an object about the size of Mars colliding with an Earth close to the end of its formation (accretion process) yields an iron-poor moon with an appropriate earthmoon system mass and angular momentum.

The intent of this research was to understand how various initial conditions affect the outcomes of large bodies that collide. We also wished to clarify how the 
initial conditions affect satellite and ring formation. Pedagogically, this research provided opportunities for undergraduate physics majors to apply Newtonian physics in a computational setting, encouraged them to extend the method to more realistic scenarios and engage in parallel computing.

We emphasize that since the bodies involved in the simulations are crudely constructed due to a limited number of particles available, the results should be interpreted generally. Hence the drive of this work is not to accurately reflect the structures of real planets but to rather get an understanding of the general physical principles present in planetary collisions.

\section{COMPUTATIONAL APPROACH}

In the work presented here a deterministic algorithm to simulate the motion of the bodies that model the planets is used. Initially there are two planets (P1 and P2) composed of N1 and N2 spherical particles, respectively, such that $\mathrm{N} 1+\mathrm{N} 2=\mathrm{N}$, the total number of particles in the simulation. Each particle (i) has mass $\mathrm{m}_{\mathrm{i}}$ and radius $r_{i}$. $P 1$ and $P 2$ are formed by isolated equilibration: placing their constituent particles loosely packed within imaginary spheres not interacting with each other and subsequently letting time advance with algorithm described later. The centers of P1 and P2 are separated by a displacement $\mathrm{R}_{0 x} \hat{i}+\mathrm{R}_{0 \mathrm{y}} \hat{j}$ and $\mathrm{P} 2$ is given an initial velocity $\vec{V}_{0}$ such that $\mathrm{P} 1$ will strike $\mathrm{P} 2$. Particle $(i)$ experiences a gravitational force

$$
\vec{F}_{i}=\sum_{j=1}^{N} \frac{G m_{i} m_{j}}{r_{i j}^{2}} \hat{r}_{i j}\left(1-\delta_{i, j}\right) .
$$

Here G is Newton's universal gravitational constant and the $m_{i}$ are the fragments' masses. Although not used in the simulations reported here, the particles composing the planets may also experience forces from electric fields

$$
\vec{F}^{i}{ }_{e}=q_{i} \vec{E}_{e x t}-\sum_{j=1}^{N} \frac{k q_{i} q_{j}}{r_{i j}^{2}} \hat{r}_{i j}\left(1-\delta_{i, j}\right) .
$$

as well as from magnetic fields

$\vec{F}^{i}{ }_{m}=$

$q_{i} \vec{v}_{i} \times\left(\vec{B}_{e x t}+\sum_{j=1}^{N} \frac{\mu_{0}}{4 \pi} \frac{q_{j} \vec{v}_{j} \times \vec{r}_{i j}}{r_{i j}^{3}}\left(1-\delta_{i, j}\right)\right)$.

Here $\vec{F}_{e}^{i}$ and $\vec{F}_{m}^{i}$ are the electric and magnetic forces on particle (i), respectively; $\vec{E}_{\text {ext }}$ and $\vec{B}$ ext are the external electric and magnetic fields, respectively; the $q_{i}$ are the particles' charges, $\mathrm{k}$ is Coulomb's constant and $\mu_{0}$ is the permeability of free space. After the forces on all $\mathrm{N}$ particles are calculated, the system is advanced in time:

$$
\begin{aligned}
& \vec{r}_{i}(t+\Delta t)=\vec{r}_{i}(t)+\vec{v}_{i}(t) \Delta t+\frac{1}{2} \frac{\vec{F}_{i}}{m_{i}}(\Delta t)^{2} \\
& \vec{v}_{i}(t+\Delta t)=\vec{v}_{i}(t) \Delta t+\frac{\vec{F}_{i}}{m_{i}} \Delta t .
\end{aligned}
$$

The time-step in the simulation is chosen such that the overlap of any two-planet fragments is small compared to either of their dimensions. After the system has advanced, the algorithm sorts through all $\mathrm{N}$ particles and determines which groups of particles were initially touching and are now overlapping because of attractive forces and which groups of particles were not initially touching and are now overlapping because of true collisions.

The former class of particles is referred to as a touching group and its constituents are dealt with in the following way. The algorithm determines the number of touching groups and which particles are in each touching group. When examining each touching group, its center of mass as well as center of mass velocity is calculated. Then all particles (i) and (j) in each touching group which are touching are separated along their relative displacement from (i) to (j) $\vec{r}_{i j}$ until they are no longer overlapping; subsequently the center of mass of that particular touching group is re-adjusted so as to equal its value before the touching group was expanded.

The second class of particles is comprised of those that are overlapping 
because they are involved in some true collision. A particle may collide with a group of particles already touching. In that case, the algorithm expands all the particle pairs (i,j) that are touching along $\vec{r}_{i j}$ and then treats the collision as a series of virtual collisions which propagates by involving consecutive pairs of masses $(i, j)$ overlapping in that group in a sequence:

$$
\begin{aligned}
& \vec{v}_{i}{ }^{\prime}=\vec{v}_{i}+\left(\frac{(1+e) m_{j}}{m_{i}+m_{j}}\right) \frac{\left(\vec{v}_{i j} \bullet \vec{r}_{i j}\right)\left(\vec{r}_{j}-\vec{r}_{i}\right)}{r_{i j}^{2}} \\
& \vec{v}_{j}{ }^{\prime}=\vec{v}_{j}-\left(\frac{(1+e) m_{i}}{m_{i}+m_{j}}\right) \frac{\left(\vec{v}_{i j} \bullet \vec{r}_{i j}\right)\left(\vec{r}_{j}-\vec{r}_{i}\right)}{r_{i j}^{2}} .
\end{aligned}
$$

Here $\vec{v}_{i j}$ is the velocity of particle (j) relative to that of (i) and $e$ is the elasticity of the collision, which can be taken to range anywhere from $e=1$ to $e=0.1$; $e=0.8$ is used in this work. The system may not advance in time unless the configuration is reached where a virtual collision does not result in particle overlap; such a configuration is taken to be the real outcome of the collision. It is well known that uncertainty in the outcome of the simulation may arise when more than two particles collide and touch at once, which could also be the case [11]. Since the algorithm searches for touching pairs, virtual collisions still proceed at different times when three or more bodies collide at once. Such artificial delay introduces uncertainty in the outcome of the simulation so in order to alleviate this effect the post-collision velocities are calculated as averages of the velocities obtained when the algorithm searches once in forward order and once in reverse order.

The method used was validated by testing it in situations involving simple geometries where the results are known: one- and two- dimensional arrays of interacting and non-interacting masses undergoing collisions of varying elasticity.

The algorithm was also parallelized by implementation of MPI, message-passing interface, to decrease the time taken to run the simulation. The MPI is used to transfer the relevant data from one machine to any number of other machines on the network. The algorithm divides up the force calculations equally between the machines and each machine advances the particles assigned to it in time. The new slices of velocity and position arrays are then broadcast to the other machines. By implementing the MPI on a system of 2000 particles the time taken to run is decreased by about $1 / N$, where $N$ is the number of processors, if $\mathrm{N}$ was less than 4 . As $\mathrm{N}$ continues to increase the time taken to run the process still decreases, but the ideal $1 / \mathrm{N}$ relation no longer holds. Further detailed computation scaling studies are warranted.

\section{DISCUSSION CONCLUSIONS}

AND

a. Collisions of Two Planets with Large Dense Central Masses (partially formed planets)

The two initial planets $\mathrm{P} 1$ and $\mathrm{P} 2$ are each composed of 250 planetesimals. $\mathrm{P} 1$ contains one planetesimal with a radius of $6 \times 10^{-5}$ Astronomical Units (AU) and a mass of $10^{-3} \mathrm{M}_{\mathrm{SUN}}$ and 249 planetesimals each with a radius of $10^{-5} \mathrm{AU}$ and a mass of $10^{-5} \mathrm{M}_{\text {SUN. }}$ P2 contains one planetesimal with a radius of $6 \times 10^{-5} \mathrm{AU}$ and a mass of $10^{-}$ ${ }^{3} M_{\text {SUN }}$ and 249 planetesimals each with a $10^{-5} \mathrm{AU}$ radius and a mass of $2 \times 10^{-5} \mathrm{M}_{\mathrm{SUN}}$. The common structure of P1 and P2 is that of a large central sphere covered in a uniform thin layer made of the smaller planetesimals-the layer being two planetesimals thick or less. The initial position of $P 1$ was $x_{1}=0 A U, y_{1}=0 A U$, and $z_{1}=0 \mathrm{AU}$, with a velocity $\mathrm{v}_{\mathrm{x} 1}=0 \mathrm{AU} / \mathrm{yr}, \mathrm{v}_{\mathrm{y} 1}=0$ $A U / y r, v_{z 1}=0 A U / y r$. The initial position of $P 2$ was $x_{2}=10^{-4} \mathrm{AU}, y_{2}=4^{*} 10^{-5} \mathrm{AU}, z_{2}=0 \mathrm{AU}$, with a velocity of $v_{x 2}=-10 A U / y r, v_{y 2}=0$ $\mathrm{AU} / \mathrm{yr}, \mathrm{v}_{\mathrm{z} 2}=0 \mathrm{AU} / \mathrm{yr}$. Upon collision $\mathrm{P} 1$ and P2 were offset by $25 \%$ of the value of their radii. Prior to the collision there was a shift form potential energy to kinetic energy because of the acceleration of the planets due to gravity.

Since the collisions are inelastic the total energy of the system decreases as time runs. The largest decrease in energy occurs when P1 and P2 initially collide, but energy is always lost because of collisions between the individual planetesimals. Figure 1 illustrates the energy history for a typical 
collision, including curves for the total kinetic energy

$$
T=\frac{1}{2} \sum_{i=1}^{N} m_{i} v_{i}^{2}
$$

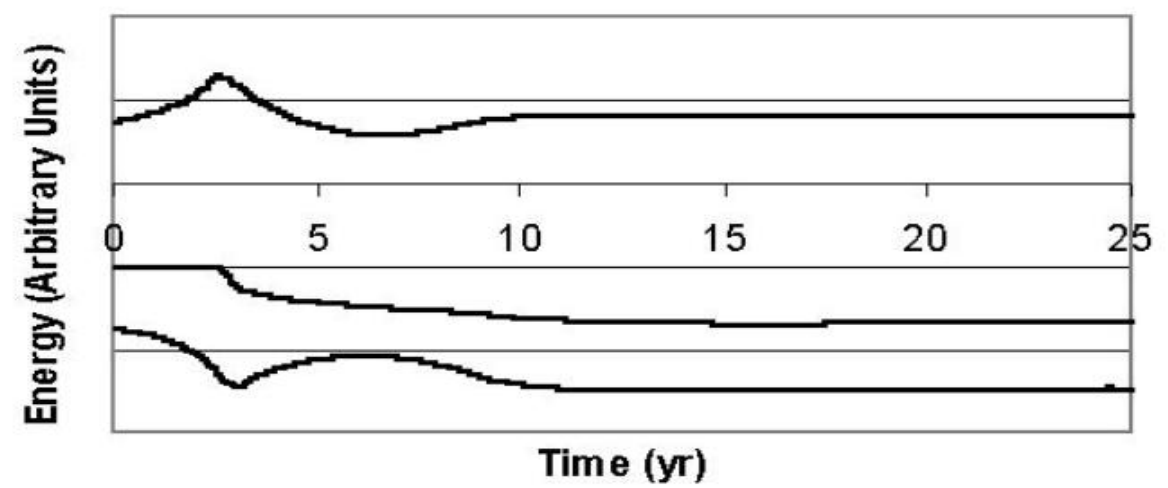

Figure 1. History of potential energy $\mathrm{V}$ (lowest curve), kinetic energy $\mathrm{T}$ (upper curve) and total energy $E$ (middle curve) through the first collision discussed.

and the total potential energy

$$
V=-\sum_{i=1}^{N} \sum_{j=i+1}^{N} \frac{G m_{i} m_{j}}{r_{i j}},
$$

The total mechanical energy $E=T+V$. Upon collision $a$ few of the planetesimals with $r=10^{-5} \mathrm{AU}$ were ejected, but the vast majority of the planetesimals were held together by gravity and formed a single slowly rotating body, that was nearly spherical as the smaller planetesimals filled in the area between the two large planetesimals. After $25 \mathrm{yrs}$ all of the ejected particles were traveling in nearly straight paths and there was no indication of any of these any longer showing the possibility of an orbit, though some had completed partial orbits before they escaped the gravity of the central mass. A series of ray-traced rendering of simulations with these initial conditions appears in Figure 2.

The previous simulation was then repeated only with $\mathrm{y}_{2}$ changed to $8 \times 10^{-5} \mathrm{AU}$. This produced a collision that was $50 \%$ offset. The results were similar to the previous simulation but only more small planetesimals were ejected and more partial orbits were formed before all partial capture orbits were extinguished. Also the rotation rate of the new planet formed was greater. The previous simulation was then repeated for $y_{2}=1.2 \times 10^{-4} \mathrm{AU}$. This also produced systems similar to those obtained with the earlier initial configurations.

In the next simulation $\mathrm{y}_{2}=1.6 \times 10^{-4}$ AU immediately after the collision the system is very similar to the earlier systems only that it is rotating much faster. As time progresses this fast rotation continually causes the lightest planetesimals mass of $10^{-5} \mathrm{M}_{\mathrm{SUN}}$ to be ejected from the planet, and there are also planetesimals of $2 \times 10^{-5} \mathrm{M}_{\mathrm{SUN}}$ ejected occasionally. The rotation of the planet also prevents it from obtaining the nearly spherical shape of the earlier planets, because it causes the small planetesimals that aren't ejected to remain evenly distributed on the surface of the large planetesimals. The continual distortion of the object as it rotates might serve as an ejecta pumping mechanism and, in reality, an energy dissipation mechanism. This constant ejecta forms a cloud of over 100 hundred planetesimals around the planet. In this cloud some of the planetesimals are gravitationally bound to each other and eventually form small touching groups of two or three particles. Also almost all of the 
planetesimals in the cloud are rotating to some extent since they were rotating as they left and this leads to many possible orbits. It cannot be confirmed that a stable orbit is ever formed because this simulation could only be run out to 125 years. This is because this simulation is very computationally demanding and only a 

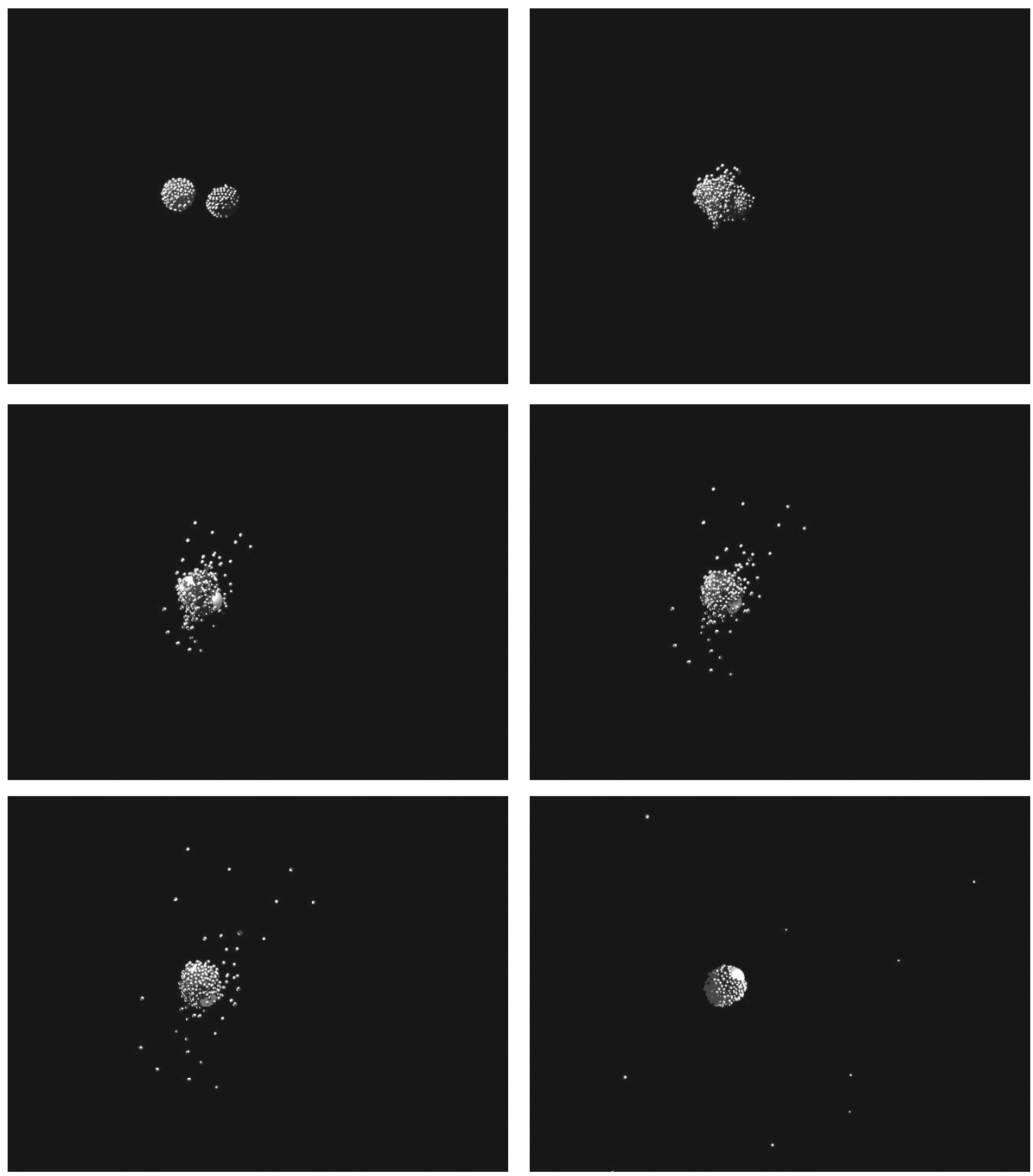

Figure 2. Ray-traced renderings of the first simulation discussed, sequentially from upper left to lower right. There is a time gap between the fifth and sixth images in order to show the final outcome $50 \mathrm{yr}$. after the initial impact. Most of the ejecta from the collision are lighter masses, although a few heavier ones are thrown off as well. The final object formed is rotating and the heavy central cores are clearly visible.

limited amount of time could be used to run it. It took over two weeks of computer time to run to the 125-year simulation. It is also hard to simplify the system by replacing the main planet with a single large planetesimal, because the latter is constantly breaking up. Ray-traced renderings of the fastest rotating system are shown in Figure 3. For a final simulation $y_{2}$ was set to $2 \times 10^{-4} \mathrm{AU}$. The collision was such that the only the outer layer consisting of the small planetesimals were the only spheres directly involved in the collision. This collision caused the least amount of planetesimals to be ejected on 
the initial collision. Seven of these eight planetesimals traveled away in straight path and never indicated an orbit but only made a partial orbit. The planet that resulted from this collision was initially very similar to the previous one. It was rotating fast and this
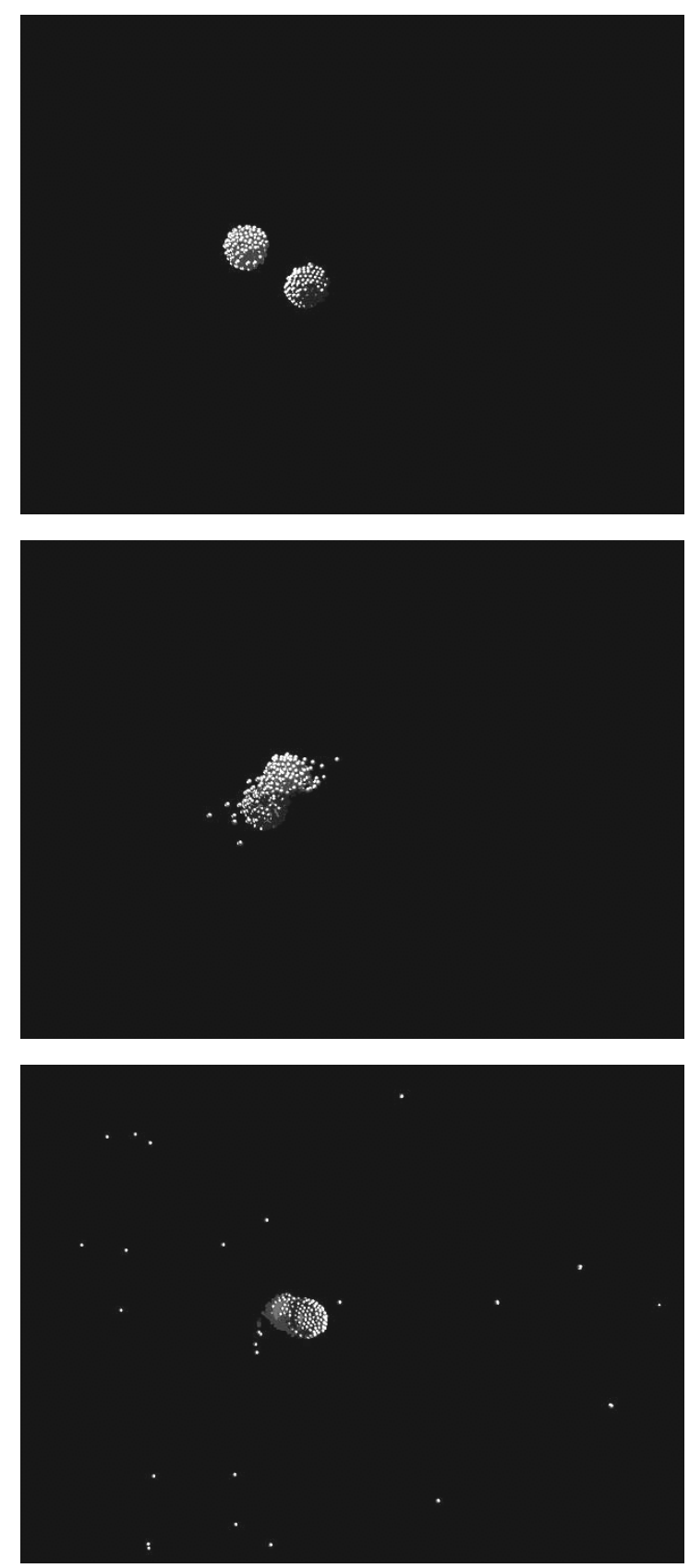

rotation was preventing the planet from obtaining a spherical shape. As time progressed it was clearly much different than the previous simulation because it never ejected any planetesimals and was able to maintain its elongated shape.
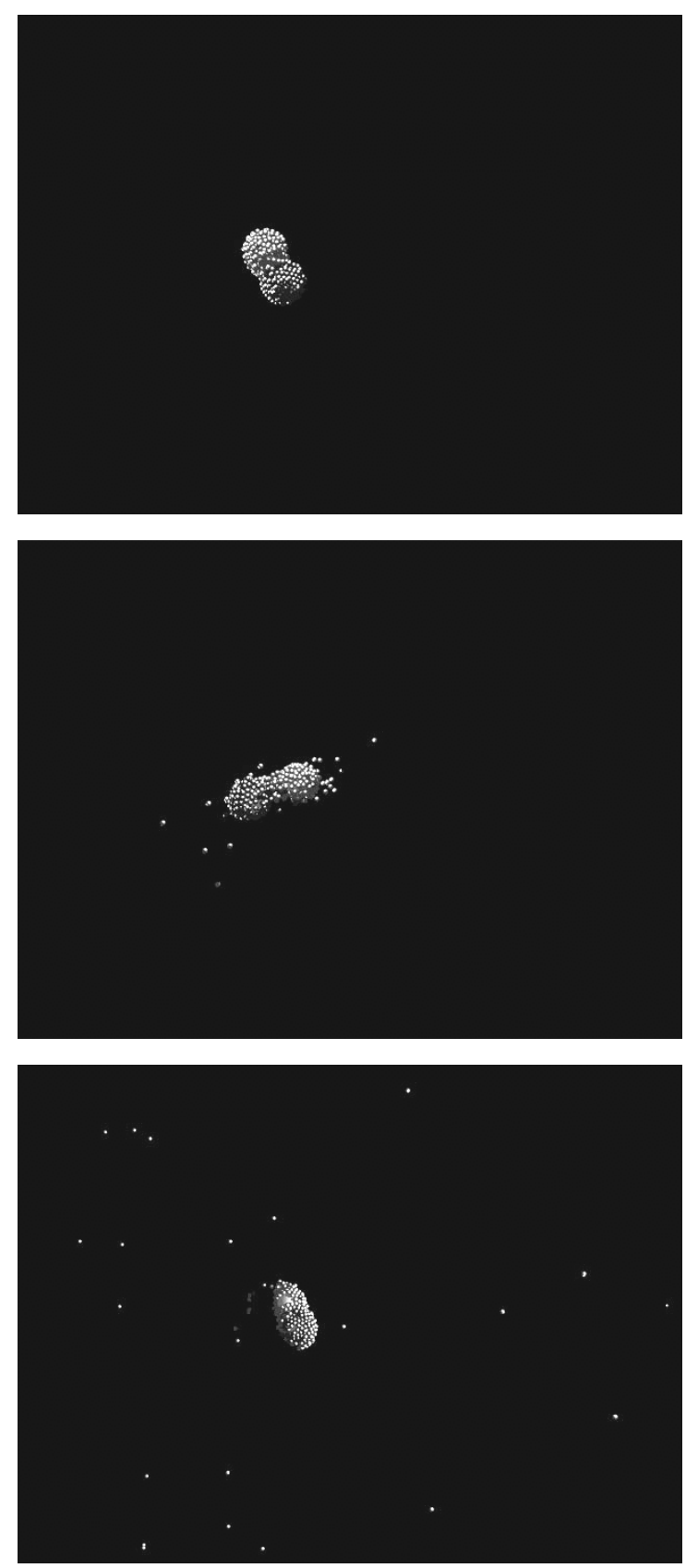

Figure 3. Ray-traced renderings of the series of simulations yielding maximum rotation of the system after collision. There is a gap in time between the fourth and fifth frames; the last one is $50 \mathrm{yr}$. after the impact. Rotation and mass ejection are noticeable after the collision and most of the ejecta in this sequence are lighter masses. 

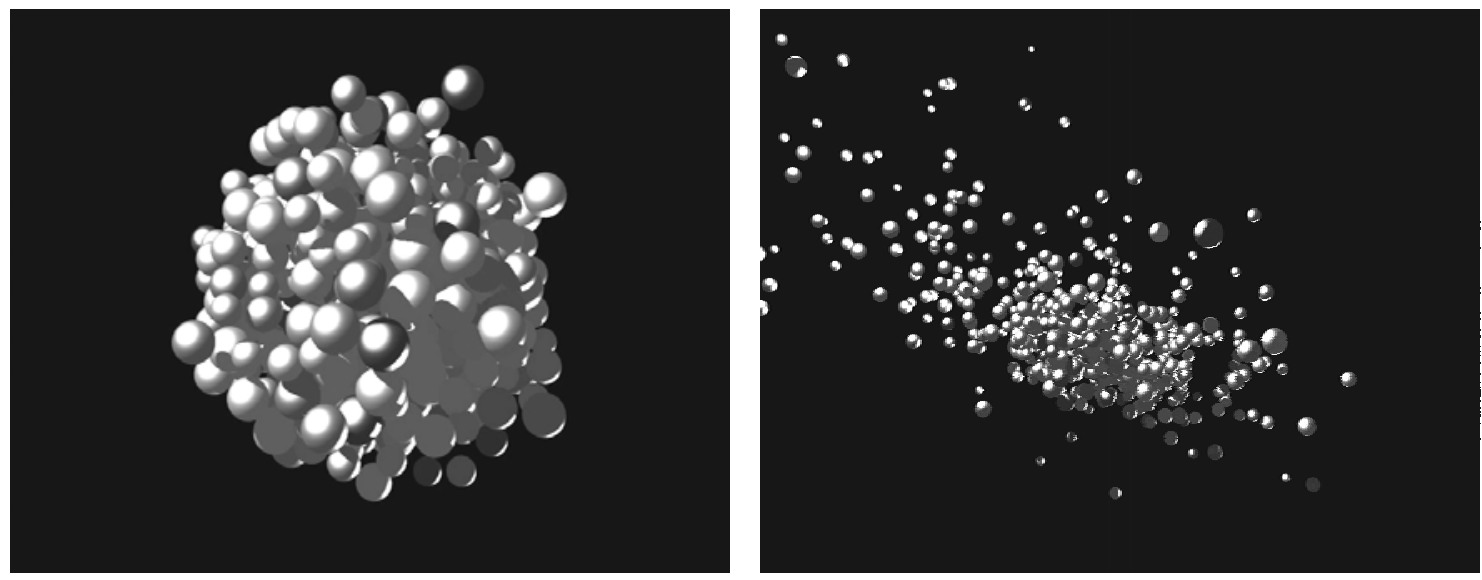

Figure 4. Ray-traced renderings of results with the initial larger planet formed from planet fragments of varying densities (left) and the system after collision (right). The sizes shown are to scale and the colors range from a low density of 5,050 $\mathrm{M}_{\mathrm{SUN}} / \mathrm{AU}^{3}$ (darkest color) to a high density of $13,4666 \mathrm{M}_{\text {SuN }} / \mathrm{AU}^{3}$ (lightest color).

b. Collision Of Planets Consisting of Planetesimals of Varying Densities (Very Young Protoplanets)

P1 consists 500 planetesimals of varying densities that have a maximum density of $13,4666 \mathrm{M}_{\text {SuN }} / \mathrm{AU}^{3}$. The planetesimals varied in radius from $4 \times 10^{-5}$ $\mathrm{AU}$ to $6 \times 10^{-5} \mathrm{AU}$. P2 consists of 100 planetesimals of the same radius with densities between 5,050 $\mathrm{M}_{\mathrm{SUN}} / \mathrm{AU}^{3}$ and $13,4666 \mathrm{M}_{\text {Sun }} / \mathrm{AU}^{3}$. The initial position of $\mathrm{P} 1$ was again at the origin and the initial position of $P 2$ was $x_{2}=7.5 \times 10^{-4} \mathrm{AU}, \mathrm{y}_{2}=5 \times 10^{-}$

$A U, z_{2}=0 A U$. With an initial velocity of $\mathrm{vx}_{2}=-2 \mathrm{AU} / \mathrm{yr}$. $\mathrm{P} 1$ and $\mathrm{P} 2$ have a similar structure; in the center of the planets are the planetesimals of the highest densities. The mass of a planetesimals varies with its distance from the planetary center, with the mass decreasing as this distance increases. During the collision P2 strikes P1 such that the center of $\mathrm{P} 2$ is at the outer edge of $\mathrm{P} 1$. The collision ejected some of the mass near the impact. Here P2 had enough energy that it could escape the gravity of $\mathrm{P} 1$; the planetesimals of $\mathrm{P} 2$, though mostly broken apart traveled away from $\mathrm{P} 1$ with a small amount of the planetesimals from P1. Upon impact a wave-like disturbance emanates in $\mathrm{P} 1$ from the point of impact. This wave traveled through $\mathrm{P} 1$ and caused it break apart in multiple directions. With no central mass both $\mathrm{P} 1$ and $\mathrm{P} 2$ were unable to reform into single planets after the collision. The lack of the large central masses also meant that there wasn't enough of a gravitational field to create any orbits. Ray-traced renderings of the initial configuration (large planet) and the system after the collision are shown in Figure 4.

\section{c. Future and Continuing Work}

The foremost effort for the future is to run the simulations with more particles so that we will be able to more finely define the colliding bodies. We again stress that, although some general dynamical phenomena are noticeable in the simulations presented here, they do not yield accurate models of what may happen when real planets collide. For example, the model should contain planet fragments of different masses, sizes and shapes. In addition this work models fairly large objects but it is desirable to model collisions between objects of the sizes seen in our solar system or calculated for new extrasolar planets. Such size scaling will be meaningful only when the resolution mentioned earlier is achieved. Presently we are also seeking to model planetary breakup due to gravitational tides as a component in the mechanism of ring formation, in addition to more realistically simulating properties of the planets involved in the collision (composition structure, thermal stresses, impact stresses, etc.) Also conducting runs using other integration schemes than the one chosen and introducing better methods 


\section{ACKNOWLEDGEMENTS}

The authors would like to thank the University of Northern lowa for providing the first author with an undergraduate summer fellowship as well as a SOAR grant during which time the work reported here was completed.

\section{REFERENCES}

1. Marcus, Philip S., Press, William $\mathrm{H}$. and Teukolsky, Saul A. Stablest Shapes for an Axisymmetric Body of Gravitating, Incompressible Fluid. The Astrophysical Journal, Vol. 214, p 584-597 (1977).

2. Stemwedel, Sally W., Yuan, Chi and Cassen, Patrick. Equilibrium models for self-gravitating inviscid disks resulting from the collapse of rotating clouds. The Astrophysical Journal, Vol. 351, p 206221 (1990).

3. Williams, Harold A. and Tohline, Joel E. Circumstellar ring formation in rapidly rotating protostars. The Astrophysical Journal, Vol. 334, p 449-464 (1988).

4. Boss, Alan Paul. Protostellar formation in rotating interstellar clouds II. Axially symmetric collapse. The Astrophysical Journal, Vol. 237, p 563-573 (1980).

5. Regev, O. and Shaviv, G. Formation of protostars in collapsing, rotating, turbulent clouds. The Astrophysical Journal, Vol. 245, p 934-959 (1981).

6. Tohline, Joel E. Ring formation in rotating protostellar clouds. The Astrophysical Journal, Vol. 236, p 160171 (1980).

7. Bills, Bruce G. Venus: Satellite orbital decay, ephemeral ring formation, and subsequent crater production. Geophysical Research Letters, Vol. 19, p 1025-1028 (1992).

8. Ipatov, S. Computer modeling of he evolution of plane rings of gravitating particles moving around the sun. Soviet Astronomy, Vol. 25, p 617-623 (1981).

9. Bu-lin, Liu. Ring formation and its evolution in a disc of rotating protostellar clouds. Acta Astronomica Sinica, Vol. 25, p 156-167 (1984).

10. Canup, Robin M. and Asphaug, Erik. Origin of the Moon in a giant impact near the end of the Earth's formation. Nature Vol. 412, p 708-712 (2001); and references therein.

11. M.P. Allen and D.J. Tildesley, Computer Simulation of Liquids (Oxford University Press, New York City) 1989.

\section{University of Northern lowa}
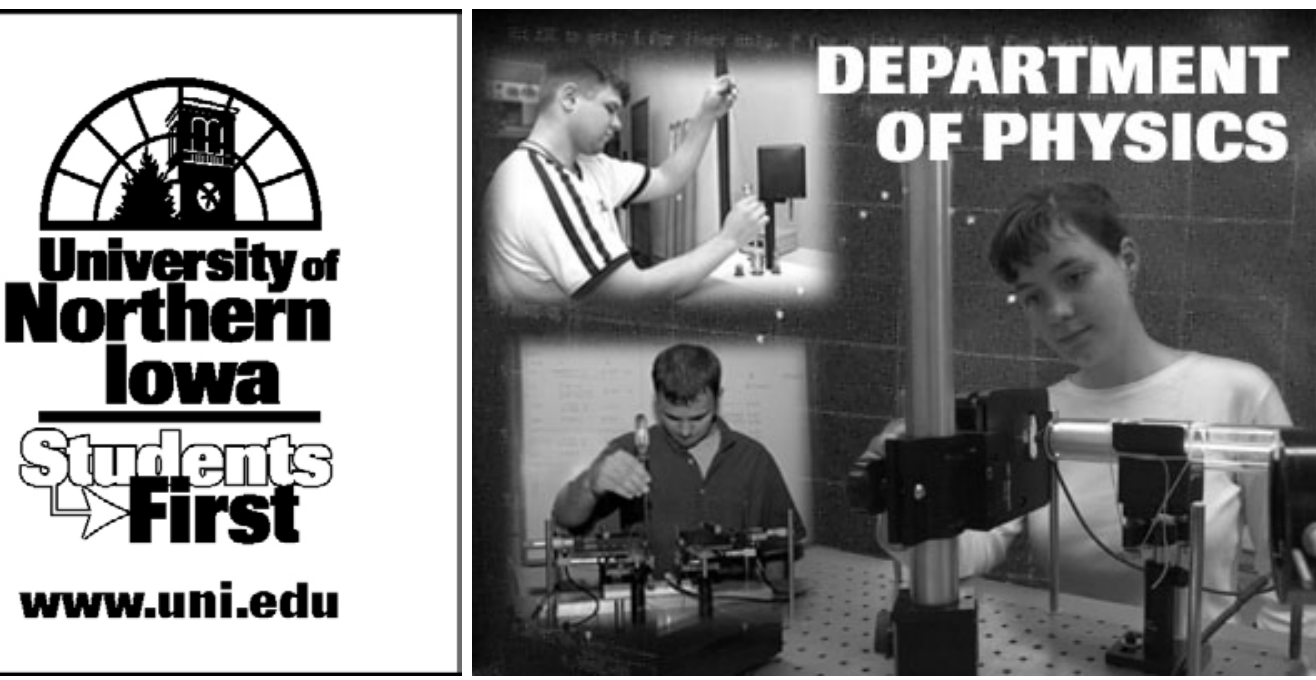